\title{
Re-evaluation of the Gottfried sum using neural networks
}

\author{
Riccardo Abbate ${ }^{1}$ and Stefano Forte F,2 $^{1,2}$ \\ ${ }^{1}$ Dipartimento di Fisica, Università di Milano \\ ${ }^{2}$ INFN, Sezione di Milano, Via Celoria 16, I-20133 Milano, Italy
}

(Dated: August 1, 2021)

\begin{abstract}
We provide a determination of the Gottfried sum from all available data, based on a neural network parametrization of the nonsinglet structure function $F_{2}$. We find $S_{G}=0.244 \pm 0.045$, closer to the quark model expectation $S_{G}=\frac{1}{3}$ than previous results. We show that the uncertainty from the small $x$ region is somewhat underestimated in previous determinations.
\end{abstract}

PACS numbers: $13.60 . \mathrm{Hb}, 11.55 . \mathrm{Hx}$

The Gottfried sum

$$
S_{G}\left(Q^{2}\right) \equiv \int_{0}^{1} \frac{d x}{x}\left[F_{2}^{p}\left(x, Q^{2}\right)-F_{2}^{n}\left(x, Q^{2}\right)\right]
$$

provides a determination of the light flavor asymmetry of the nucleon sea. The discovery by the NMC [1, 2, 3 ] that $S_{G}$ deviates from the simple quark model expectation $S_{G}=\frac{1}{3}$ has provided first evidence for an up-down asymmetry of the nucleon sea, a finding which has been subsequently confirmed in different contexts, is routinely included in modern parton fits, and has spawned a large theoretical literature [4, 5]. Because the scale dependence of the Gottfried sum is known up to next-to-next-to leading order [6, 7] its precise determination is potentially interesting for tests of QCD and the determination of the strong coupling.

The experimental determination of a sum rule, and especially of the associated uncertainty, is nontrivial because structure function data are only available at discrete values of $x$ and in general not all given at the same $Q^{2}$. Therefore, one needs interpolation and extrapolation in $x$ in order to cover the full range $0 \leq x \leq 1$, and extrapolation in $Q^{2}$ in order to bring all data at the same $Q^{2}$. Also, it is not obvious how to combine data from different experiments without losing information on experimental errors and correlations.

In Refs. 8, 9] the NNPDF collaboration has proposed a method for the parametrization of structure functions and parton distributions, and has constructed a parametrization of the proton, deuteron, and nonsinglet $F_{2}^{\mathrm{NS}} \equiv F_{2}^{p}-F_{2}^{n}$ structure functions based on all available experimental information, including experimental and theoretical uncertainties and their correlation. This parametrization has been recently used by various authors [10, 11, 12] as an unbiased interpolation of existing data.

Here, we wish to provide a determination of the Gottfried sum based on this parametrization, which, in the nonsinglet case, relevant for the Gottfried sum, is based on the structure function data from the NMC 13] as well as those from the BCDMS collaboration 14, 15, which are rather more precise and cover a different kinematic region (see Fig. 1).

The parametrization of Refs. [8, 9] provides a Monte Carlo sample of replicas of the structure function for all $x$ and $Q^{2}$, so the Gottfried sum and associated error can be straightforwardly determined by integrating over $x$ at fixed $Q^{2}$, and averaging over the sample. The error on the parametrization blows up when extrapolating outside the measured region, so that the region where reliable predictions are obtained can be inferred from the parametrization itself.

First, we compare to the result of Refs. 2, 3], where the contribution to the Gottfried integral Eq. (11) from the measured region $0.004<x<0.8$ at $Q^{2}=4 \mathrm{GeV}^{2}$ is determined to be $S_{G}\left(0.004<x<0.8,4 \mathrm{GeV}^{2}\right)=$ $0.2281 \pm 0.0065$ (stat.) \pm 0.019 (syst.) $=0.2281 \pm 0.020$. The previous determination of Ref. [2], based on about half the statistics, had $S_{G}\left(0.004<x<0.8,4 \mathrm{GeV}^{2}\right)=$ $0.221 \pm 0.008$ (stat.) \pm 0.019 (syst.) $=0.2281 \pm 0.021$. Using the neural parametrization of Ref. [8] we get

$$
S_{G}\left(0.004<x<0.8,4 \mathrm{GeV}^{2}\right)=0.2281 \pm 0.0437,
$$

where the error includes statistical and (correlated) systematic uncertainties which are combined in the parametrization of Ref. 8] (NNPDF result, henceforth). Despite the (accidental) perfect agreement with NMC of

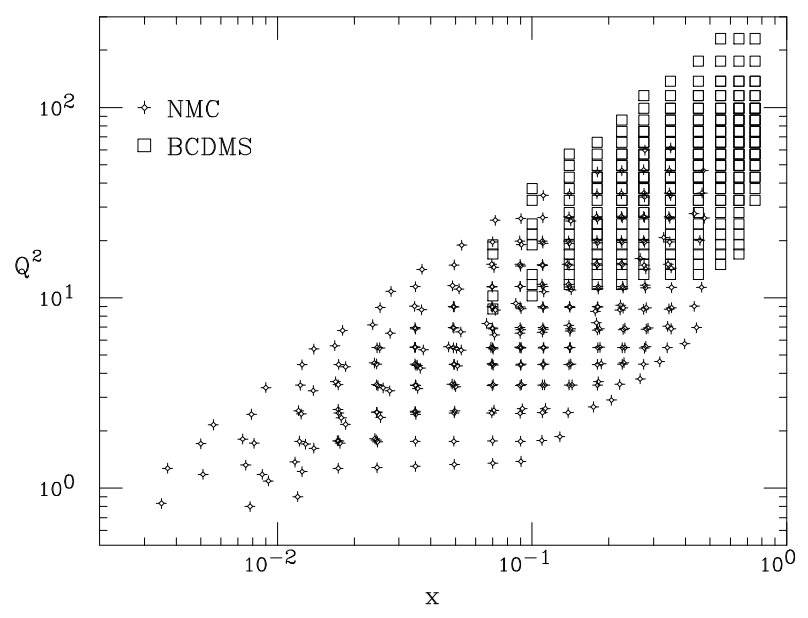

FIG. 1: Data used to construct the neural network parametrization of $F_{2}^{\mathrm{NS}}$ of Ref. [8]. 
the central value of the NNPDF result Eq. (2), the uncertainty is more than twice as large. This is suprizing, in view of the fact the data used in neural parametrization include both NMC and BCDMS, and cover a wider kinematic region.

In fact, the dataset used by $\mathrm{NMC}$ in their determination of the $F_{2}^{p} / F_{2}^{d}$ ratio which is used to compute the Gottfried sum in Ref. [3] is about four times as large as that used by the same collaboration in their structure function determination of Ref. 13 which was used to construct the neural network parametrization [8], essentially because the detrmination of a structure function ratio allows more generous cuts than the absolute determination of the structure function. However, it is unclear whether this can explain the higher precision of the determination of Ref. [3], given that the error is dominated by systematics, and the determination of $S_{G}$ requires anyway knowledge of at least one structure function on top of the structure function ratio.

TABLE I: The contribution to the Gottfried sum at $Q^{2}=$ $4 \mathrm{GeV}^{2}$ from the region $x_{\text {min }} \leq x \leq 0.8$ as obtained by NMC 2] and with neural networks. The error is only statistical for NMC, while it is the total combined statistical and systematic uncertainty for NNPDF. The total NMC systematics on $S_{G}(0.004 \leq x \leq 0.8)$ is equal to 0.019 .

\begin{tabular}{|c||c|c||}
\hline \multicolumn{1}{|c||}{$x_{\min }$} & \multicolumn{2}{c|}{$S_{G}\left(x_{\min }<x<0.8\right)$} \\
\hline & NMC & NNPDF \\
\hline \hline 0.004 & $0.221 \pm 0.008$ & $0.2281 \pm 0.0437$ \\
\hline 0.010 & $0.213 \pm 0.005$ & $0.2378 \pm 0.0273$ \\
\hline 0.020 & $0.203 \pm 0.004$ & $0.2334 \pm 0.0232$ \\
\hline 0.040 & $0.183 \pm 0.004$ & $0.2157 \pm 0.0217$ \\
\hline 0.060 & $0.171 \pm 0.003$ & $0.1985 \pm 0.0202$ \\
\hline 0.100 & $0.149 \pm 0.003$ & $0.1693 \pm 0.0169$ \\
\hline 0.150 & $0.125 \pm 0.003$ & $0.1398 \pm 0.0133$ \\
\hline 0.200 & $0.107 \pm 0.003$ & $0.1154 \pm 0.0107$ \\
\hline 0.300 & $0.074 \pm 0.003$ & $0.0761 \pm 0.0074$ \\
\hline 0.400 & $0.047 \pm 0.002$ & $0.0460 \pm 0.0052$ \\
\hline 0.500 & $0.025 \pm 0.002$ & $0.0241 \pm 0.0035$ \\
\hline 0.600 & $0.012 \pm 0.002$ & $0.0102 \pm 0.0019$ \\
\hline \hline
\end{tabular}

In order to understand this state of affairs, in Table \we compare the contribution to the Gottfried integral Eq. (11) from the measured experimental region $x_{\min } \leq x \leq 0.8$ [2] with that obtained from neural networks. The NNPDF determination uncertainty includes statistical and correlated systematic errors, while the NMC experimental result Ref. 2] only determines the overall systematic uncertainty. Combining the total NMC systematics (which is highly correlated between bins) with the statistical error of Table \ the NNPDF and NMC total uncertainties are seen to be in very good agreement up to the next-to-smallest $x$ bin. However, when the smallest $x$ bin is included the NNPDF uncertainty almost doubles, while the NMC uncertainty (which is dominated by systematic) is essentially unchanged.

This suggests that the NMC uncertainty from the smallest $x$ bin might be underestimated. The reason for this is understood by inspection of Fig. 2, where the NNPDF and NMC determinations of the nonsinglet structure function at $Q^{2}=4 \mathrm{GeV}^{2}$ are compared. Note that the NMC error bars are purely statistical, and that the NNPDF error band has high point-to-point correlation (so the error on $S_{G}$ is much smaller than the spread of the integrals of the one-sigma curves). Note also that the NMC data points 2 2 are obtained by combining their determination of $F_{2}^{d}$ and of the ratio $F_{2}^{p} / F_{2}^{d}$, and extrapolating the results at fixed $x$ to a common $Q^{2}$, while the NNPDF results are obtained [8] by interpolating and extrapolating the full set of NMC and BCDMS $F_{2}$ data: hence the two determinations should agree within errors, but they are not expected to be on top of each other.

The two determinations are indeed seen to be in good agreement. The agreement of the total uncertainty on $S_{G}$ for $x \gtrsim 0.01$ proves that, once systematics is included, the total uncertainties also agree. At the smallest $x$ values, $x \lesssim 0.01$, the uncertainty on $F_{2}$ blows up nonlinearly as a function of $x$, due to the lack of smaller $x$ data which could constrain the extrapolation. The NNPDF result, which is obtained integrating $F_{2}^{\mathrm{NS}}$ reproduces this blowup. The NMC, based on summing over bins (i.e. multiplying the value at the bin center by the bin width) implicitly assumes that the error is linear across the bin and thus underestimates the error on the last bin.

We conclude that the NMC error on the Gottfried sum from the measured region is smaller than the NNPDF error Eq. (2) entirely due to the contribution of the smallest $x$ bin, and that this in turn is largely due to the fact that the sum over bin by NMC underestimates the non-

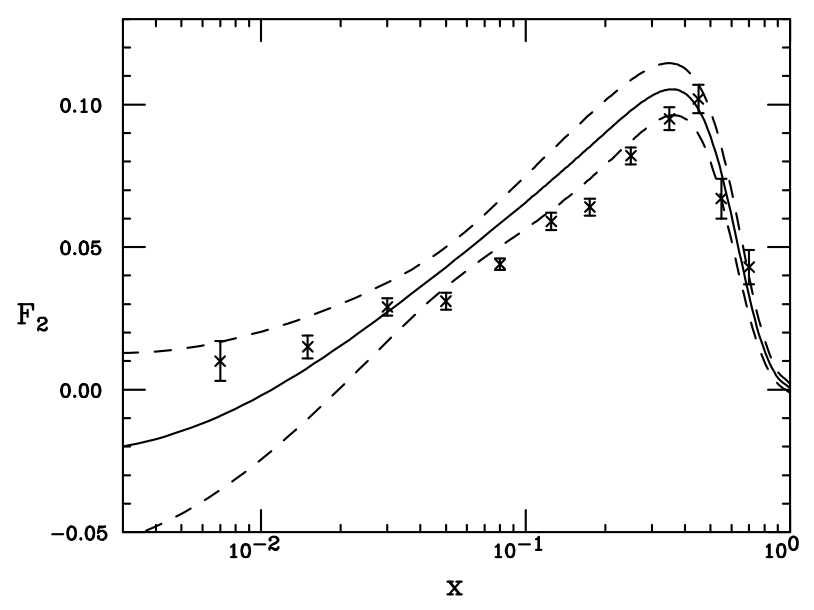

FIG. 2: The nonsinglet structure function $F_{2}^{\mathrm{NS}}\left(x, Q^{2}\right)$ as a function of $x$ at $Q^{2}=4 \mathrm{GeV}^{2}$. The solid line is the central value obtained from neural network and the dashed lines give the corresponding one-sigma error band (note errors are highly correlated betwen different values of $x$ ). The experimental points are from Ref. [2], the error bars are statistical only. 
linear growth of the uncertainty at the edge of the data region [22].

Let us now turn to the best determination of $S_{G}$ that can be obtained from neural networks. To this purpose, we note that even though in principle the neural parametrization of $F_{2}$ provides a value for all $x$ and $Q^{2}$, when extrapolating outside the data region this determination becomes unreliable: the uncertainty grows rapidly, but eventually the uncertainty itself is unreliable. Furthermore, whereas the neural nets do satisfy the kinematic constraint $F 2\left(1, Q^{2}\right)=0$, they do not satisfy the theoretical constraint $F 2\left(0, Q^{2}\right)=0$, so the error on $S_{G}$ would diverge if the sum rule were determined by simply integrating from 0 to 1 . This is as it should be, because the $x \rightarrow 0$ region corresponds to the limit of infinite energy, and thus it is even in principle experimentally unaccesible: the associate error is therefore infinite unless one makes some theoretical assumption.

Therefore, we determine the Gottfried sum by integrating in $x$ at fixed $Q^{2}$ for $x_{\min } \leq x \leq 1$, and adding to this integral a contribution from the small $x$ region determined by extrapolation. Note that no extrapolation is necessary in the large $x$ region, because the coverage of the large $x$ region from the BCDMS data together with the kinematical constraint at $x=1$ are sufficient to pin down the structure function with good accuracy at large $x$ [8].

The small $x$ extrapolation requires theoretical assumptions. In Ref. [1], it was assumed that $F_{2}\left(x, Q^{2}\right) \underset{x \rightarrow 0}{\sim} A x^{b}$, and the constants $A$ and $b$ were determined by fitting to the smallest $x$ data. However, the assumption that the small- $x$ power behaviour has already set in in the smallest measured $x$ bins does not seem justified. Indeed, in the singlet case the small $x$ behaviour observed at HERA is not seen in the NMC data and canot be predicted by them [9, 16]. Also, on theoretical grounds one would expect the asymptotic small $x$ behaviour to set in around $x \approx 10^{-3}[17$. Hence, fitting the small $x$ exponent to the data might lead to an underestimate of the uncertainty on the small $x$ extrapolation, if the exponent $b$ comes out too large.

TABLE II: Determination of the Gottfried sum with neural networks. The scale is given in $\mathrm{GeV}^{2}$. The contribution from $x<x_{\min }$ is obyained by extrapolation and given $100 \%$ uncertainty (see text).

\begin{tabular}{|c|c|c|c|}
\hline$Q^{2}$ & $x_{\min }$ & $S_{G}\left(x_{\min }<x<1\right)$ & $S_{G}$ \\
\hline \hline 1 & 0.007 & $0.2566 \pm 0.0773$ & $0.2849 \pm 0.0917$ \\
\hline 2 & 0.005 & $0.2522 \pm 0.0389$ & $0.2548 \pm 0.0494$ \\
\hline 3 & 0.007 & $0.2430 \pm 0.0299$ & $0.2479 \pm 0.0454$ \\
\hline 4 & 0.008 & $0.2380 \pm 0.0302$ & $0.2415 \pm 0.0477$ \\
\hline 5 & 0.008 & $0.2330 \pm 0.0340$ & $0.2329 \pm 0.0507$ \\
\hline 10 & 0.01 & $0.2246 \pm 0.0428$ & $0.2278 \pm 0.0627$ \\
\hline 30 & 0.008 & $0.2395 \pm 0.0632$ & $0.2450 \pm 0.0860$ \\
\hline \hline $1.5-4.5$ & 0.006 & $0.2438 \pm 0.0320$ & $0.2438 \pm 0.0449$ \\
\hline
\end{tabular}

Therefore, we extrapolate by assuming that the struc- ture function at small $x$ displays the behaviour predicted by Regge theory [18] $F_{2}\left(x, Q^{2}\right) \underset{x \rightarrow 0}{\sim} A \sqrt{x}$ : even if in actual fact this behaviour were to set in at smaller $x$, we would only be miscalculating the contribution to the integral from the matching region. Of course, we cannot exclude non-Regge behaviour at small $x$, but if the Gottfried integral Eq. (11) exists at all, its integrand is unlikely to diverge much stronger than $\frac{1}{\sqrt{x}}$ at small $x$. Note that the small $x$ behaviour found by fitting to the data in Ref. 2] is in fact somewhat softer than this, namely $F_{2}\left(x, Q^{2}\right) \underset{x \rightarrow 0}{\sim} A x^{0.59}$.

Hence, for all $x<x_{\min }$ we take $F_{2}\left(x, Q^{2}\right)=$ $F_{2}^{s x}\left(x, Q^{2}\right)$, with

$$
F_{2}^{s x}\left(x, Q^{2}\right)=A \sqrt{x}
$$

We fix the normalization coefficient $A$ by matching this behaviour to the neural network result at a somewhat larger value of $x$. This enables us to match at a value of $x$ which is inside the data region, while only using $F_{2}^{s x}\left(x, Q^{2}\right)$ Eq. (3) at the edge or outside the data region itself. In practice, we match at $x=1.5 x_{\mathrm{min}}$; we have checked that results change very little if we move the matching point from $1.1 x_{\min }$ to $2 x_{\min }$. Matching to the neural network determination of $F_{2}\left(1.5 x_{\min }, Q^{2}\right)$ gives us a one-sigma error band $A=A_{\text {match }} \pm \sigma_{A}$.

The contribution to $S_{G}$ from $x<x_{\min }$ is determined as the integral of $F_{2}^{s x}\left(x, Q^{2}\right)$ computed with $A=A_{\text {match }}$. This is given $100 \%$ uncertainty within the one-sigma error band of $A$, namely, the extrapolation error on is taken to be equal to the integral of $F_{2}^{s x}\left(x, Q^{2}\right)$ computed with $A=\left|A_{\text {match }}\right|+\sigma_{A}$. The contribution to $S_{G}$ is then added to the contribution from the measured region, while the corresponding errors are added in quadrature.

For each value of $Q^{2}$ we can thus find the value of $x_{\text {min }}$ which minimizes the total uncertainty. There is a tradeoff in that if $x_{\min }$ is raised, the error on the measured region

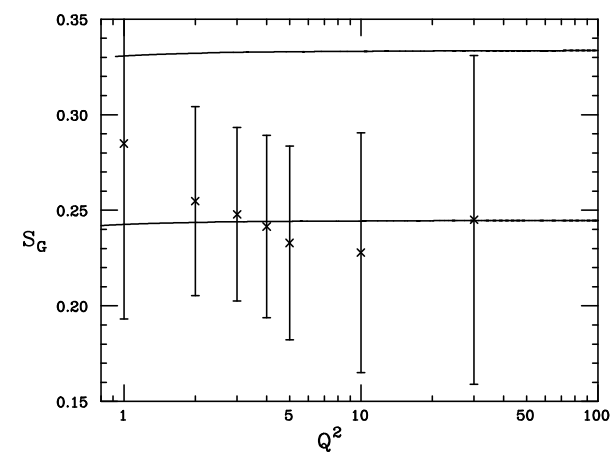

FIG. 3: The value of the Gottfried sum $S_{g}$ (as given in table III) as a function of the scale. The curve shows the scale dependence computed in perturbative QCD at NNLO [6, 7]: upper curve assuming the quark model value $S_{G}(\infty)=\frac{1}{3}$, lower curve assuming our best-fit $S_{G}\left(1 \leq Q^{2} \leq 5\right)=.245$. 
decreases rapidly, but there is an increase in size of the small $x$ extrapolation, which is $100 \%$ uncertain. The results for the Gottfried sum $S_{G}$ Eq. (11), the contribution from the measured region, and the value of $x_{\min }$ which minimizes the error are shown in Table II.

In Fig. 3 these values are compared to the NNLO perturbative prediction for the scale dependence [6, 7], [23] computed with the assumption that as $Q^{2} \rightarrow \infty$ the naive quark model value $S_{G}=\frac{1}{3}$ is reproduced. This shows that, even though the uncertainty in our determination is rather larger, and the central value somewhat closer to the quark model prediction than the SMC value [3], the quark model value and hence flavour symmetry of the light quark sea are somewhat disfavoured.

It is apparent from Fig. 3 that the predicted perturbative dependence of $S_{G}$ is very slight, and it is in fact entirely negligible on the scale of the error on $S_{G}$. For example, the increase in $S_{G}$ from $Q^{2}=1 \mathrm{GeV}^{2}$ to $Q^{2}=10 \mathrm{GeV}^{2}$ due to perturbative evolution is less than $1 \%$. Hence, we may exploit the fact that neural networks retain full information on correlations to combine the determination of $S_{G}$ at different values of $Q^{2}$. When correlations are fully taken into account, this can be done by computing $S_{G}$ at an increasingly large number of values of $Q_{\min }^{2} \leq Q^{2} \leq Q_{\max }^{2}$, until the result doesn't change as the number of values of $Q^{2}$ at which $S_{G}$ is calculated in the given interval does not change: because the correlation of $S_{G}\left(Q_{1}^{2}\right)$ and $S_{G}\left(Q_{2}^{2}\right.$ tends to one as $Q_{1} \rightarrow Q_{2}$, adding new points eventually stops bringing in new information.

However, because $S_{G}$ is very highly correlated between different values of $Q^{2}$, and the uncertainty increases quite fast when the scale is moved to low $Q^{2}$ (where data uncertainties are larger) or high $Q^{2}$ (where there is little data coverage at small $x$ ), the uncertainty on this averaged determination is only marginally smaller than any of those which we obtained at fixed $Q^{2}$. Optimizing both the $Q^{2}$ range and the choice of $x_{\min }$ we get our best value

$$
S_{G}\left(1.5 \leq Q^{2} \leq 4.5 \mathrm{GeV}^{2}\right)=0.244 \pm 0.045
$$

which can be taken to hold for any $Q^{2}$ in the given range. The NNLO $Q^{2}$ dependence of this result (assumed to hold at $Q^{2}=3 \mathrm{GeV}^{2}$ ) is also displayed in Fig. 3

A more precise determination of the Gottfried sum will only be possible once more data will become available, such as those which could be obtained injecting deuterons in HERA [16], from a high-energy upgrade of JLAB [19], or from future facilities, such as the Electron-Ion collider 20] or a neutrino factory 21].
[1] P. Amaudruz et al. (New Muon), Phys. Rev. Lett. 66, 2712 (1991).

[2] M. Arneodo et al. (New Muon), Phys. Rev. D50, 1 (1994).

[3] M. Arneodo et al. (New Muon), Nucl. Phys. B487, 3 (1997), hep-ex/9611022.

[4] S. Forte, Phys. Rev. D47, 1842 (1993).

[5] S. Kumano, Phys. Rept. 303, 183 (1998), hep$\mathrm{ph} / 9702367$.

[6] A. L. Kataev and G. Parente, Phys. Lett. B566, 120 (2003), hep-ph/0304072.

[7] D. J. Broadhurst, A. L. Kataev, and C. J. Maxwell, Phys. Lett. B590, 76 (2004), hep-ph/0403037.

[8] S. Forte, L. Garrido, J. I. Latorre, and A. Piccione, JHEP 05, 062 (2002), hep-ph/0204232.

[9] L. Del Debbio, S. Forte, J. I. Latorre, A. Piccione, and J. Rojo (NNPDF), JHEP 03, 080 (2005), hep$\mathrm{ph} / 0501067$.

[10] M. Osipenko et al. (CLAS), Phys. Rev. D67, 092001 (2003), hep-ph/0301204.

[11] S. Simula and M. Osipenko, Nucl. Phys. B675, 289 (2003), hep-ph/0306260.

[12] G. Corcella and L. Magnea, Phys. Rev. D72, 074017 (2005), hep-ph/0506278.

[13] M. Arneodo et al. (New Muon), Nucl. Phys. B483, 3 (1997), hep-ph/9610231.

[14] A. C. Benvenuti et al. (BCDMS), Phys. Lett. B223, 485 (1989).

[15] A. C. Benvenuti et al. (BCDMS), Phys. Lett. B237, 592 (1990).

[16] M. Dittmar et al. (2005), hep-ph/0511119.
[17] B. I. Ermolaev, M. Greco, and S. I. Troyan, Phys. Lett. B622, 93 (2005), hep-ph/0503019.

[18] R. K. Ellis, W. J. Stirling, and B. R. Webber, $Q C D$ and collider physics, vol. 8 (1996).

[19] S. I. Alekhin, A. L. Kataev, S. A. Kulagin, and M. V. Osipenko, Nucl. Phys. A755, 345 (2005), hep-ph/0502107.

[20] A. L. Deshpande, Nucl. Phys. Proc. Suppl. 105, 178 (2002).

[21] M. L. Mangano et al. (2001), hep-ph/0105155.

[22] The final NMC determination [3] is obtained from a dataset which is about a factor 2 larger than that of Ref. 2], but the bin-by-bin determination of $S_{G}$ shown in Tab. I is not available for these data. However, the conclusion of the analysis of table $\prod$ and figure 2 based on the data of Ref. 2] are unchanged because the uncertainty in the smallest $x$ bins is largely dominated by systematics. Note also that at small $x$ the determination of $F_{2}^{\mathrm{NS}}$ with the method of Refs. 1, 2, 3], based on the measurement of the $F_{2}^{p} / F_{2}^{d}$ ratio, is a priori more precise than that based on the determination of their difference because in the smallest $x$ bins there is a large cancellation so that $F F_{2}^{\mathrm{NS}}$ is about one order of magnitude smaller than $F_{2}^{p}$ and $F_{2}^{d}$. Nevertheless, this is the case essentially for all $x$, while the NNPDF and NMC errors only disagree in the smallest $x$ bin.

[23] Note that the numerical value of the NNLO $n_{f}$ independent contribution is given incorrectly due to typos in Ref. [6]: the correct value is given in Ref. [7] when $n_{f}=3$ and it is equal to -0.850 instead of -0.809 when $n_{f}=4$. 